\title{
Persistent Map Saving for Visual Localization for Autonomous Vehicles: An ORB-SLAM 2 Extension
}

\author{
Felix Nobis*, Odysseas Papanikolaou, Johannes Betz and Markus Lienkamp \\ Chair of Automotive Technology, Technical University of Munich \\ Munich, Germany \\ Email: *nobis@ftm.mw.tum.de
}

\begin{abstract}
Electric vhicles and autonomous driving dominate current research efforts in the automotive sector. The two topics go hand in hand in terms of enabling safer and more environmentally friendly driving. One fundamental building block of an autonomous vehicle is the ability to build a map of the environment and localize itself on such a map. In this paper, we make use of a stereo camera sensor in order to perceive the environment and create the map. With live Simultaneous Localization and Mapping (SLAM), there is a risk of mislocalization, since no ground truth map is used as a reference and errors accumulate over time. Therefore, we first build up and save a map of visual features of the environment at low driving speeds with our extension to the ORBSLAM 2 package. In a second run, we reload the map and then localize on the previously built-up map. Loading and localizing on a previously built map can improve the continuous localization accuracy for autonomous vehicles in comparison to a full SLAM. This map saving feature is missing in the original ORB-SLAM 2 implementation.
\end{abstract}

We evaluate the localization accuracy for scenes of the KITTI dataset against the built up SLAM map. Furthermore, we test the localization on data recorded with our own small scale electric model car. We show that the relative translation error of the localization stays under $1 \%$ for a vehicle travelling at an average longitudinal speed of $36 \mathrm{~m} / \mathrm{s}$ in a feature-rich environment. The localization mode contributes to a better localization accuracy and lower computational load compared to a full SLAM. The source code of our contribution to the ORB-SLAM2 will be made public at: https:/github.com/TUMFTM/orbslammap-saving-extension.

Index Terms-Simultations Localization and Mapping, Localization, Re-localization, Autonomous Vehicles, SLAM, Map, ORB-SLAM 2

\section{INTRODUCTION}

Mastering autonomous driving is an ongoing challenge in current research in the automotive industry and academia. In order to navigate safely, an autonomous vehicle needs to build an accurate representation of its environment and estimate its position within it. In this work, we aim to localize a vehicle along a previously driven and mapped route. It serves as an intermediary development for the application as a localization method in circuit racing scenarios [1]. In these conditions, the vehicle needs to be able to localize its position precisely to enable maneuvering at high velocities.

An effective measure for estimating the ego vehicle position is the use of a global navigation satellite system (GNSS). However, the accuracy of these systems is limited. Noise is introduced due to the atmospheric conditions, signal reflections and clock errors. Real Time Kinematic (RTK) positioning systems can reduce these errors by relying on the correction signal of a fixed calibrated base station. On the downside, such systems are reliant on further infrastructure and come with additional costs [2].

To be independent of GNSS or RTK coverage for localization, vehicles use lidar, camera and radar sensors to perceive the environment. On the basis of these sensor data, a map representation is computed for the current environment. Simultaneously, the vehicle localizes itself relatively to this map (SLAM). When these local maps are saved at a fixed global GNSS position, vehicles driving in previously mapped locations only need to localize themselves on the given map to get a global position estimation. During a SLAM, a localization error can lead to a discontinuity in the map and thereby hinder any further localization or re-localization. The localization-only mode saves computational resources and enables re-localization in the case of a previous localization error.

In the following, we adapt the ORB-SLAM 2 [3] algorithm to such two-stage localization-only scenarios. In a first step, we create a map of the environment at 
low speeds through a SLAM. In the second step, we load the map and localize the vehicle on it at higher speeds. We show that the computational load and the localization error can be lowered in this localization-only strategy. We briefly cover related research and review the algorithm we build upon in Section III. Section III discusses our implementation of the ORB-SLAM 2 extension. We evaluate our extension in different scenarios in Section $[\mathrm{IV}$ and close the paper with our conclusions.

\section{RELATED WORK}

The various onboard sensors for localization - radar, camera and lidar - have individual strengths and drawbacks.

[4] present a localization approach on a previously builtup map of radar measurements. The localization error they show is small in terms of lateral positioning, as the features coming from the roadside are distinctive. The error in longitudinal direction is greater since the features are less distinctive in this direction.

The field of lidar SLAM is widely studied in literature [5]-[7]. Lidar sensors are widely available in research, especially for indoor robotics. The reliant range measurements give a relatively dense estimation of the environment compared to radar systems. In the open source community, the Adaptive Monte Carlo Localization (AMCL) implementation in the Robot Operating System (ROS) framework is widely used for localization on a 2D occupancy grid, and is applied successfully to a racing scenario by [8].

In comparison to radar and lidar data, camera data is more dense and can perceive textures or features on straight walls more easily. It is therefore the sensor with the most potential for localization. Rangan [9] builds up a local map with camera features and associates them with GNSS coordinates. During localization, their system matches the features of the current camera frame against a GNSS-queried subset of the map features. In contrast, we build up a global map which contains all extracted features of the environment. After defining an initial GNSS location for the map, we rely on the stereo camera information only.

\section{A. Visual SLAM}

An extensive overview of current visual SLAM approaches is given in [10]. One difficulty in working with camera sensors for localization purposes is that of depth generation from images. With the knowledge of the camera intrinsic parameters, we can calculate the direction of the light beam that resulted in every pixel in the image. However, the distance from which this light originated and thereby the real world 3D position of the sensed object cannot be measured. In recent years, approaches have been developed to estimate the depth from images of monocular cameras with learning methods [11]-[13]. However, these do not yet possess the same quality as the direct depth calculation from a stereo camera system, which we rely on in this work.

Two state-of-the-art open source visual SLAM systems are ORB-SLAM 2 [3] and Direct Sparse Odometry (DSO) [14]. Those two systems are representatives of the two main approaches for visual odometry systems: Feature-based methods and direct methods. ORBSLAM 2 matches sparse visual features (keypoints and descriptor vectors) between image frames in order to perform the tracking, mapping and place-recognition tasks. DSO minimizes the photometric error of consecutive images in pixel values and does not rely on feature points. Both of these methods have proven their accuracy in the KITTI Vision Benchmark [15]. Direct methods have gained popularity over the last years due to their high accuracy, efficiency and robustness [16]. In this paper, the focus is on evaluating the relocalization accuracy of SLAM systems when a map is already available and pose estimation is challenging, e.g. at high velocities. DSO is a purely visual odometry system which does not perform loop closure and cannot recover its position by re-localizing if tracking is lost. We develop our persistent map saving and loading extension to ORB-SLAM 2 which has the ability to re-localize the ego vehicle on previously seen frames, using feature extraction and matching.

\section{B. ORB-SLAM 2}

In this section, we review some of the workings of the ORB-SLAM 2 algorithm which are of importance to the proposed extension in this paper.

Internally, ORB-SLAM 2 consists of three main parallel threads: Tracking, local mapping and loop closure. The tracking thread localizes the camera with every frame by finding feature matches and minimizing the reprojection error of these features to the previous frame. In parallel, the local mapping thread uses local bundle adjustment to optimize a window of keyframes and keypoints. The loop closing thread is responsible for detecting revisited areas and correcting the accumulated drift using pose-graph optimization with the $g^{2} o$ framework [17]. After the pose-graph optimization, it launches a fourth thread that performs full bundle adjustment 
and optimizes the whole map and trajectory estimations between keyframes. Global optimization is essential in SLAM systems, as even small trajectory estimation errors accumulate over time, which leads to false trajectory estimates for longer mapping sequences. By performing loop closure and global trajectory optimization, ORBSLAM 2 is able to minimize the accumulated error and improve the localization accuracy.

ORB-SLAM 2 extracts ORB features [18] from the input images to encode the environment in a sparse representation. These features are robust with regard to rotation and scale, while being fast to extract and match, thus allowing for real-time operation [19]. ORB-SLAM 2 recognizes revisited places based on the DBoW2 library [20]. It compares the current image features in a binary format against the binary features saved in the hierarchical DBoW2 database. If a certain similarity score between the current frame and a representation in the database is reached, a loop closure is detected. The functionality is used to detect loop closures and to perform re-localization in mapped areas in SLAM mode. In localization mode, the local mapping and loop closing threads are deactivated and the system uses only the tracking thread to track the camera in the previously mapped scene. This is accomplished by matching features in the current frame to the features of the previous frame, as well as by mapping points to perform re-localization.

\section{MAP SAVING EXTENSION IMPLEMENTATION}

We use the stereo camera version of the ORB-SLAM 2 ROS adaption by [21] as the base line for our extension. This section briefly describes the design of the map saving extension and the inner workings of the existing approach which had to be adapted.

Despite the fact that ORB-SLAM 2 offers the ability to run in localization mode, it does so only in online mode during the same run. The user can set the system to localization mode manually in a Graphical User Interface. However, when the SLAM process ends, the map is deleted. Therefore, we create an extended version of ORB-SLAM 2 which offers the option of saving the created map in a binary file when the SLAM process ends. After an initial mapping run and a complete system shutdown, the vehicle computer can load the saved map and localize the position of its current camera frame in it. The re-localization is performed via the localization tracking thread described above. Our extension to ORB-SLAM 2 can easily be integrated as a localization package for robots running on ROS. It can serve as an alternative package to the lidar based AMCL package or fused as an additional input to a more complex localization approach.

Regarding the implementation details, the localization mode needs to be provided with a map file that contains the objects of the ORB SLAM classes: Map, MapPoint, Keyframe and KeyframeDatabase, and the DBoW2 BoWVector and FeatureVector for each Keyframe. Therefore, we created a SaveMap method that saves this information in a binary file when the SLAM process is finalizing. For consecutive runs, the user can specify via a settings file if he wants the map file to be loaded or not. If the user decides to use a saved map, a LoadMap method is called on system startup which loads the saved Map and KeyframeDatabase and sets the system to localization mode. The latter is done automatically and there is no need for the user to manually change the mode of the system online. Both SaveMap and LoadMap methods are integrated in the System class of ORB-SLAM 2 which handles all the main functionalities. A high level flowchart of the extended version of ORBSLAM 2 can be seen in Figure 1 .

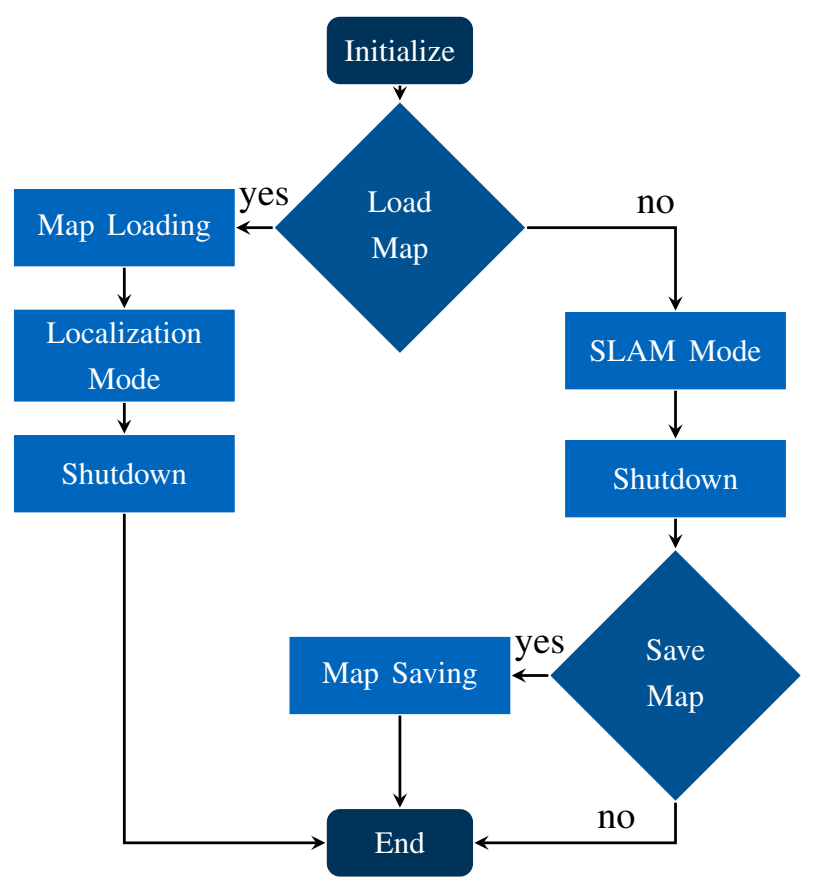

Fig. 1: Flowchart of the two operating modes: Localization (left) and full SLAM (Right) of ORB-SLAM 2.

\section{EXPERIMENTS AND RESULTS}

After introducing the used datasets and metrics in subsection IV-A, we present our method to simulate faster driving speeds using the KITTI dataset in subsection IV-B. The localization evaluation for the KITTI dataset 
is performed on a powerful desktop CPU in subsection IV-C Additionally experiments on a mobile CPU are presented in subsection IV-D for our self-recorded sequences.

\section{A. Datasets and Metrics}

We evaluate our system using the publicly available KITTI dataset [22] and recordings from a model car at 1:10 scale, built during this research project. The model car uses a ZED Stereo camera in order to acquire the visual information about its environment. We run ORBSLAM 2 on a platform which uses ROS to test the realtime performance. For the KITTI dataset, we generate ROS bags using the raw data from the Road and City categories which correspond to the Visual Odometry Sequences 01,07 and 09 recorded on motorway and urban street sections. The ground truth poses are provided and are used for the accuracy evaluation.

We record additional data at an indoor and an outdoor location at the Technical University of Munich with our model car. An example image from the outdoor street setting is shown in Figure 2. Here, the algorithm is tested in a full scale street environment without traffic. The indoor scene, depicted in Figure 3, is recorded in a the industrial hall of the Chair of Automotive Technology. In this environment, the algorithm has to operate under different lighting conditions and is presented with less distinct optical features due to passages running parallel to straight white walls.

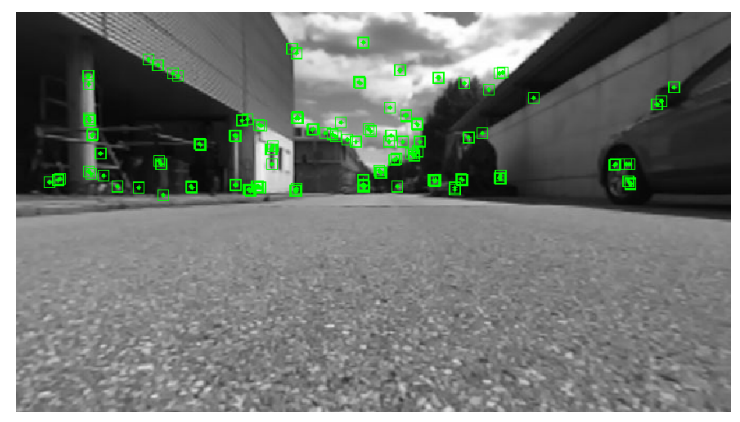

Fig. 2: Example image frame from the outdoor dataset. ORB feature locations shown in green.

In the data generated with the model car, the paths of the fast driving runs differ from the paths of the slow driving runs which are used for the map creation due to manual driving. This enables us to evaluate our persistent map saving and localization extension in the probable setting where future runs of an autonomous vehicle will have slightly different trajectories from the mapping trajectory. This adds extra difficulties to the

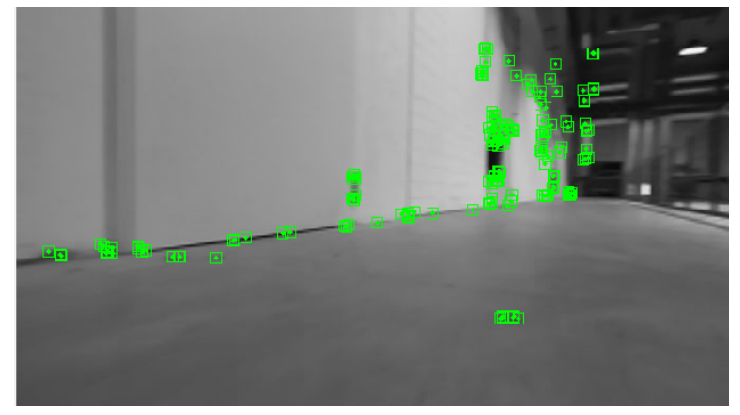

Fig. 3: Example image frame from the indoor dataset. ORB feature locations shown in green.

localization due to different perception perspective of the environment.

Before we can perform localization, we build up a map of visual features with our ORB-SLAM 2 extension. The SLAM mode is computationally demanding. Three threads are running in parallel. In the original implementation, the system reads in a stereo pair of image frames, performs the camera frame tracking and continues with the next pair of frames when the calculations are finished. Our ROS enabled version reads the image frames from a ROS bag at the same rate as they were recorded. This enables us to test the real-time capabilities of the system. The mapping run is performed at moderate driving speeds in order to ensure that ORB-SLAM 2 does not drop frames and lose track. The localization capability is then evaluated on the same sequences at higher speeds w.r.t. to the initial mapping run.

The metrics used for our evaluation are the percentage of frames where the localization failed (Lost Track: $L T$ ), the maximum consecutive time that tracking is lost $L T_{t, \max }$, the average relative translation $t_{r e l}$ and rotation $r_{r e l}$ error, as proposed in [22], as well as the absolute translation root-mean-squared error $t_{a b s}$ proposed in [23]. Tracking is lost when ORB-SLAM 2 fails to perform feature extraction and matching, and is thus unable to track the camera position between consecutive image frames. At high velocities, this can be caused by the higher rotational and translational differences of the camera viewpoints. In those cases, the algorithm can match fewer features, causing an inaccurate localization. In a scene where not many features are observable, the matching might also fail for low speeds. For our own dataset, we resort to the Lost Track metric only as there is no ground truth information available for the trajectory accuracy. 


\section{B. High Velocity Simulation for KITTI Data}

The driving speed in the KITTI data is fixed. We simulate higher driving speeds by a higher playback speed of the data. For the simulated speed, non-linearities in the vehicle dynamics might be inaccurately represented. The amount of camera frames available in the simulated dataset is greater than it would be in a recording at faster speeds. To show that the effects of these differences are minor, we record data on our model car with different driving speeds and compare the effect of the playback speed on the results. We record runs at different driving speeds in our dataset. The paths vary slightly due to the manual driving of the model car. We speed up the data of the slow runs to match the same average driving speed in slow and fast runs. The image sequences are then processed by our real-time ORB-SLAM 2 extension. We measure the percentage of camera frames where the localization fails (Lost Track) in I for those different recordings. We see that the Lost Track metric is similar for runs at high speeds as well as runs with simulated high speeds. As the compared trajectories for the indoor and outdoor category vary in their paths, the slight differences in the metric might be caused by the slightly different amount of features visible in those distinct runs. The comparable results support our choice to simulate faster driving speeds through playback speed variation for the KITTI dataset.

TABLE I: Comparison of fast driving and fast playback speeds on TUM dataset

\begin{tabular}{|c|c|c|}
\hline Sequence & Average Speed (m/s) & $L T(\%)$ \\
\hline \hline inside_fast & 6 & 1.5 \\
inside_slow-4x & 6 & 1.51 \\
\hline outside_fast & 7.2 & 0.12 \\
outside_slow-4x & 7.2 & 0.1 \\
\hline outside_fast-2x & 14.4 & 4.51 \\
outside_slow-8x & 14.4 & 3.97 \\
\hline
\end{tabular}

\section{Evaluation using the KITTI Dataset}

We evaluate the localization performance using the KITTI Visual Odometry sequences 01, 07 and 09. The algorithm runs on an Intel Xeon CPU E3-1270 v5 and 16 GB of memory.

First, we need to generate an accurate map, which represents the ground truth trajectory as closely as possible. Table II contains the evaluation results of the mapping runs on the KITTI dataset with respect to the ground truth trajectories. As the localization is performed relative to this map, any errors in the map cannot be corrected by the localization module. Sequence 01
TABLE II: SLAM accuracy relative to ground truth

\begin{tabular}{|c|c|c|c|c|c|}
\hline Seq. & $\begin{array}{c}\text { Speed } \\
(\mathrm{m} / \mathrm{s})\end{array}$ & Lost Track & $\begin{array}{c}t_{\text {rel }} \\
(\%)\end{array}$ & $\begin{array}{c}r_{\text {rel }} \\
(\% / 100 \mathrm{~m})\end{array}$ & $\begin{array}{c}t_{\text {abs }} \\
(\mathrm{m})\end{array}$ \\
\hline \hline 01 & 10 & no & 1.31 & 0.2 & 9.89 \\
\hline 07 & 6 & no & 0.51 & 0.29 & 0.52 \\
\hline 09 & 8 & no & 0.84 & 0.25 & 1.66 \\
09 & 20 & yes & - & - & - \\
\hline
\end{tabular}

consists of motorway driving with an average linear velocity of $20 \frac{\mathrm{m}}{\mathrm{s}}$. As stated in the ORB-SLAM 2 paper, the translation is difficult to estimate in this sequence because only few feature points close to the vehicle can be tracked. We reduced the playback rate of sequence 01 to $0.5 \mathrm{x}$ for the map creation which results in a more precise map. For sequence 09 a playback rate of $0.8 x$ results in the best map accuracy. A further reduction of the playback rate did not improve the accuracy results. At these slower playback speeds, the absolute translational errors for both sequences are lower than the results presented in the original ORB-SLAM 2 paper.

We also experimented with speeding up of the driving speed for the SLAM runs. Figure 4 shows the resulting trajectories for the fast and the slow SLAM runs. For the high speed run, the SLAM mode is no longer able to complete the mapping run, since the feature matching fails. This is marked in red in the figure. In the following, we show that the localization mode is still able to achieve reasonable results at those driving speeds where a SLAM fails.

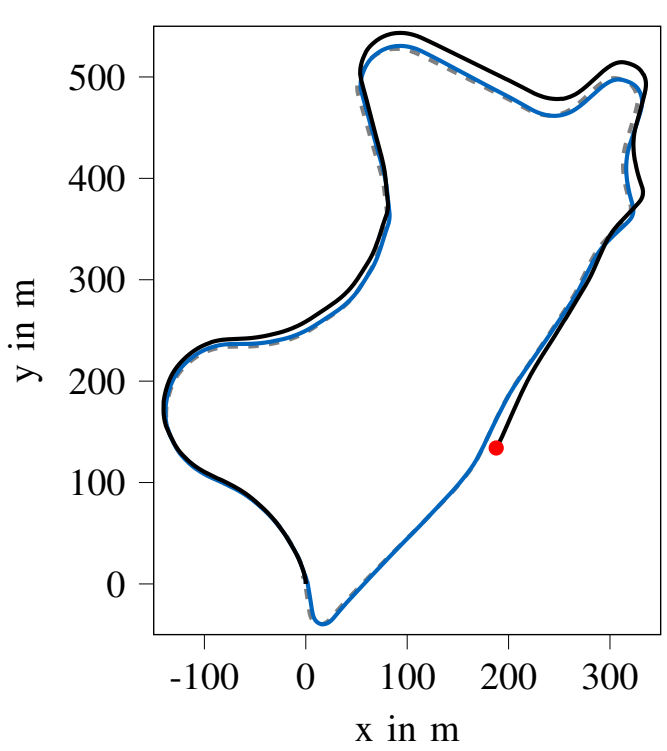

Fig. 4: KITTI Sequence 09. Ground truth (dashed gray), SLAM trajectories at $8 \frac{\mathrm{m}}{\mathrm{s}}$ (blue) and $20 \frac{\mathrm{m}}{\mathrm{s}}$ (black). The faster SLAM trajectory diverges further from the ground truth and fails at the position marked in red. 
TABLE III: Localization accuracy relative to map

\begin{tabular}{|c|c|c|c|c|c|c|}
\hline Seq. & $\begin{array}{c}\text { Speed } \\
(\mathrm{m} / \mathrm{s})\end{array}$ & $\begin{array}{c}t_{r e l} \\
(\%)\end{array}$ & $\begin{array}{c}r_{\text {rel }} \\
(\% / 100 \mathrm{~m})\end{array}$ & $\begin{array}{c}t_{a b s} \\
(\mathrm{~m})\end{array}$ & $\begin{array}{c}L T \\
(\%)\end{array}$ & $\begin{array}{c}L T_{t, \max } \\
(\mathrm{s})\end{array}$ \\
\hline 01 & 21 & 0.3 & 0.05 & 1.14 & 0 & 0 \\
01 & 37 & 4.82 & 0.31 & 10.77 & 1.9 & 0.07 \\
01 & 42 & 6.12 & 0.41 & 12.82 & 6.62 & 0.14 \\
\hline 07 & 6 & 0.04 & 0.05 & 0.1 & 0 & 0 \\
07 & 12 & 0.08 & 0.11 & 0.14 & 2.35 & 0.05 \\
07 & 18 & 0.23 & 0.13 & 0.22 & 5.87 & 0.49 \\
07 & 24 & 0.44 & 0.17 & 0.98 & 8.67 & 0.55 \\
\hline 09 & 10 & 0.03 & 0.04 & 0.01 & 0 & 0 \\
09 & 20 & 0.36 & 0.13 & 0.44 & 0.147 & 0.04 \\
09 & 25 & 0.43 & 0.16 & 0.6 & 2.15 & 0.11 \\
09 & 36 & 0.66 & 0.21 & 0.81 & 7.44 & 0.2 \\
\hline
\end{tabular}

In the next step, we localize the vehicle relative to the recorded maps at different simulated driving speeds for all sequences. The results are shown in Table III. For each sequence, we replay the trajectory at the same rate as the original recording and increase it by up to $4 x$ the original rate. At the original speed of the recording, the localization algorithm is able to localize the vehicle with errors of only a few centimeters for sequences 07 an 09 , while the error on the motorway sequence 01 is higher.

Furthermore, the results show that a reasonable localization accuracy can be achieved at speeds of up to $36 \mathrm{~m} / \mathrm{s}$ for sequence 09 . The localization approach loses track for about $8 \%$ of the frames in this case. The longest consecutive time that no localization can be performed is $0.55 \mathrm{~s}$. The presented localization system shall be integrated in a globally fused localization module with additional sensor inputs, so that such a loss rate is acceptable for the use case. The further spatial error metrics increase for higher velocities. For the sequences 07 and 09 these errors stay within a tolerable interval, especially if they are with respect to a longitudinal deviation. A mis-localization along the path is less critical than a lateral deviation in the control concept of the vehicle. For sequence 01, the spatial error metric reaches values which would make a localization impracticable. In this sequence, only few features are present for the feature matching.

In evaluating the localization algorithm, the relative error with regard to the map is most meaningful. For an application of the overall pipeline the global error with regard to the ground truth is equally important. As this paper focuses on the localization approach, the global error is shown for the sake of completeness in Table $\mathrm{V}$ in the appendix.

Various qualities of the mapping and localization algorithm are shown in the exemplary figures below. Figure 5 shows a curve where the mapping and localization results are in close alignment to the ground truth. The blue SLAM trajectory is mostly covered by the localization trajectory in the figure as their paths coincide greatly. Figure 6 shows the localization in close alignment to the reference SLAM trajectory, and confirms the good result of the localization module in sequence 09 . The global error however remains significant, as the mapping mode is not able to create an accurate global map. Figure 7 shows estimated trajectories for sequence 01 where both the mapping and the localization perform poorly due to a lack of features. Generally, it is observed that the system introduces the greatest localization errors during turns with high angular velocities. Translational movements or slow turns introduce less noise. We assume this is due to the greater feature similarity in the latter cases.

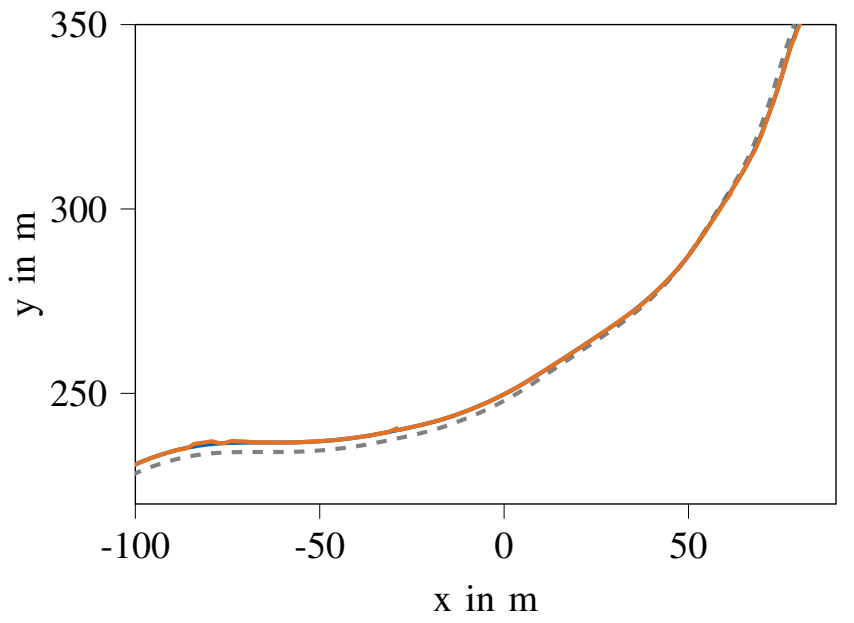

Fig. 5: KITTI sequence 09. Ground truth (dashed gray), SLAM trajectory at $8 \frac{\mathrm{m}}{\mathrm{s}}$ (blue) and localization at $36 \frac{\mathrm{m}}{\mathrm{s}}$ (orange). Both SLAM and localization align well with the ground truth.

\section{Evaluation using the Self-recorded Datasets}

Following the localization evaluation for the KITTI dataset, we evaluate the performance using our selfrecorded indoor and outdoor dataset. The trajectories at different speeds vary slightly, making for a more realistic replication of the practical use of the implementation.

In mobile robotics, whether it is self-driving cars or model cars, computational resources are a limitation factor which has to be considered. In addition to the evaluation on the hardware used in subsection IV-C, we evaluate the model car localization on the mobile NVIDIA Jetson TX2. The CPU cluster of the Jetson TX2 consists of a dual-core Denver 2 processor and a quadcore ARM Cortex-A57. In this way, we investigate the 


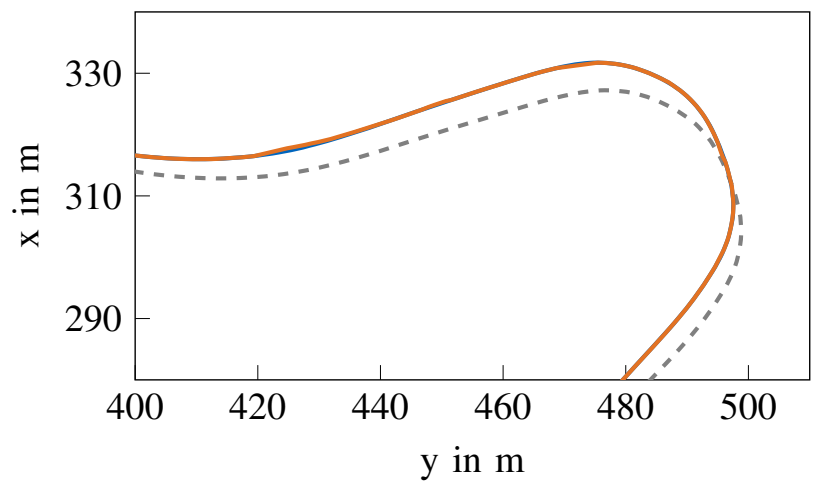

Fig. 6: KITTI sequence 09. Ground truth (dashed gray), SLAM trajectory at $8 \frac{\mathrm{m}}{\mathrm{s}}$ (blue) and localization at $36 \frac{\mathrm{m}}{\mathrm{s}}$ (orange). The SLAM trajectory has an error toward ground truth. Localization aligns well with the SLAM trajectory.

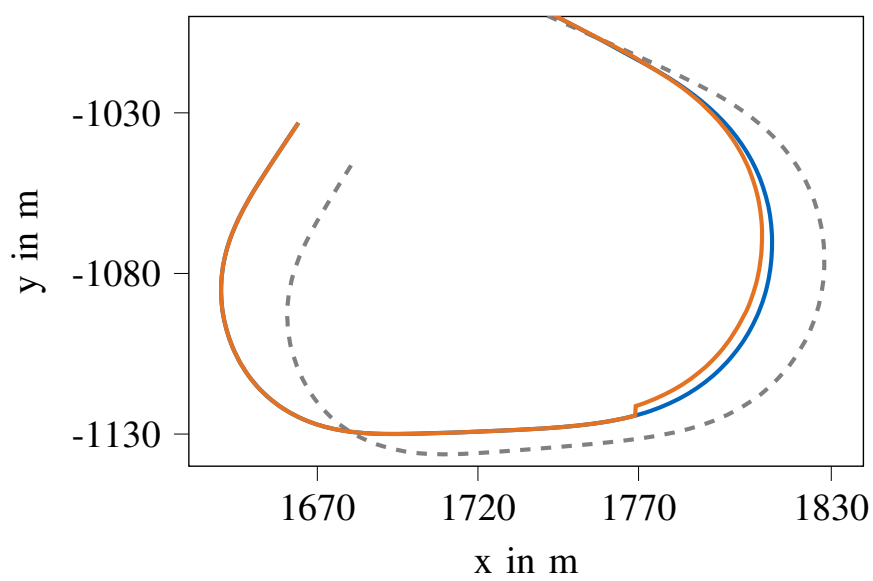

Fig. 7: KITTI sequence 01. Ground truth (dashed gray), SLAM trajectory at $10 \frac{\mathrm{m}}{\mathrm{s}}$ (blue) and localization at $42 \frac{\mathrm{m}}{\mathrm{s}}$ (orange). The SLAM trajectory has an error toward ground truth. Localization has an additional error towards the SLAM trajectory for a section of the curve.

extent to which localization accuracy is dependent on available computing power.

The SLAM mode is run using our dataset in order to create the initial maps. The trajectories of indoor and outdoors scenes are shown in Figures 8 and 9 . For the localization evaluation, only the Lost Track metric is considered since no ground truth is available.

The localization is evaluated for different speeds and trajectories against the SLAM map recorded at low speed. The localization aligns well with the SLAM trajectory for the fast computational hardware which are examplatory shown in Figures 8 and 9 . The results for the

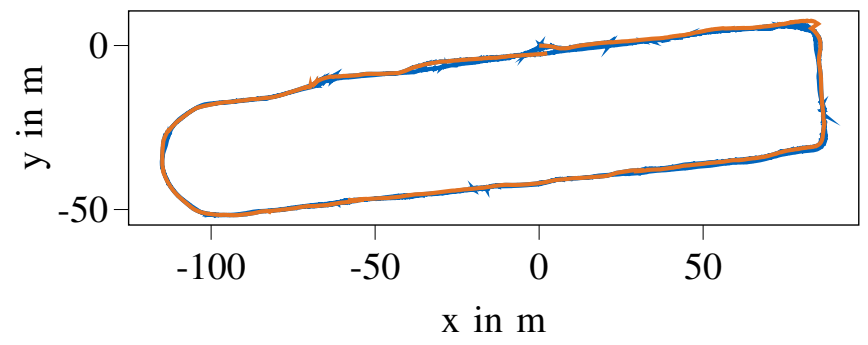

Fig. 8: TUM Outdoor scene. SLAM trajectory (blue) and localization (orange). Localization and SLAM align well.

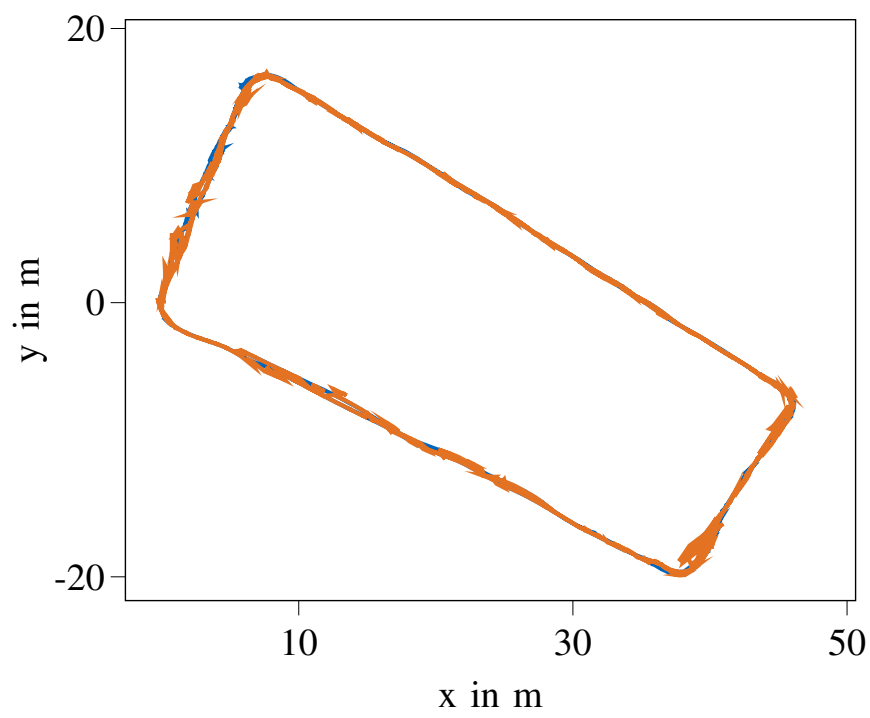

Fig. 9: TUM Indoor scene. SLAM trajectory (blue) and localization (orange). Localization and SLAM align well. We can see a drift in both trajectories as they do not form a rectangular shape, even though the recorded track is rectangular.

localization runs on the different computational devices are shown in Table IV.

TABLE IV: Localization accuracy with different computational hardware

\begin{tabular}{|c|c|c|c|c|c|}
\hline \multicolumn{2}{|c|}{} & \multicolumn{2}{|c|}{ Intel Xeon } & \multicolumn{2}{c|}{ Jetson TX2 } \\
\hline Sequence & $\begin{array}{c}\text { Speed } \\
(\mathrm{m} / \mathrm{s})\end{array}$ & $\begin{array}{c}L T \\
(\%)\end{array}$ & $\begin{array}{c}L T_{t, \max } \\
(\mathrm{s})\end{array}$ & $\begin{array}{c}L T \\
(\%)\end{array}$ & $\begin{array}{c}L T_{t, \max } \\
(\mathrm{s})\end{array}$ \\
\hline \hline inside_slow-3x & 4.5 & 0.9 & 0.21 & 6.93 & 0.65 \\
inside_fast & 6 & 1.5 & 0.31 & 10.33 & 0.54 \\
\hline outside_slow-2x & 3.6 & 0.07 & 0.04 & 7.25 & 1.68 \\
outside_fast & 7.2 & 0.12 & 0.02 & 19.23 & 1.82 \\
\hline
\end{tabular}

For the Xeon CPU, the amount of frames lost is considerably lower than the one for the NVIDIA Jetson. The embedded CPUs do not have the necessary processing power to perform the necessary feature extraction and matching in real-time. This leads to a loss of tracking 
- not due to a lack of features, but due to the real-time constraints.

On the Xeon processor the computational resources did not limit the algorithm. Here, we notice that in the indoor environment, the localization error is greater than for the outdoor scenes. This is presumably caused by the fact that the indoor environment includes areas with texture-less white walls which possess few features to be matched.

More significantly, we see that the matching fails for even lower speeds when comparing it to the results using the KITTI dataset in Section III, There are discontinuities in the estimated trajectories which can be identified as spikes in Figures 8 and 9 . The camera images of the model car are taken at a resolution of $672 \times 376$ pixels compared to the $1241 \times 376$ resolution of the KITTI dataset. Furthermore, the model car uses an input in a JPG-compressed format. The artifacts resulting from the compression are an additional challenge for the feature matching module. The necessity of good image quality for the successful implementation of the localization module is shown here.

\section{Conclusions}

This work presents an extension to a visual SLAM system via a map saving functionality. Like the original implementation, this extension enables the system to be used in slow driving conditions as a SLAM module. Additionally, it can be used in faster driving conditions as a localization module resulting in an overall more precise trajectory estimation. The system is an extension to ORB-SLAM 2 and can be used for localization in both outdoor and indoor environments. Experimental results show that it is possible to perform localization at velocities of $36 \mathrm{~m} / \mathrm{s}$ in feature-rich environments.

The localization module requires fewer computational resources than the SLAM module; however, the feature calculation and matching is demanding. We showed the limitations of the approach on an embedded CPU.

The localization does not perform well for environments with few features, such as KITTI sequence 01. This poses an open challenge to the application of such algorithms to racing scenarios where similar conditions apply. Emerging approaches use features generated by deep learning techniques [24] for SLAM applications. It is still an open research question as to whether this approach can increase the localization accuracy for the presented scenarios. At the same time, these deep features can be less variant to changing lighting conditions compared to the current optical features. This could make a re-localization possible also for different conditions such as day and night time.

The map saving extension developed and evaluated in this work improves the functionality of the original ORB-SLAM 2 approach and enables new use cases such as the application to autonomous racing for model cars.

\section{CONTRIBUTIONS AND ACKNOWLEDGEMENTS}

Felix Nobis initiated the idea of this paper and contributed essentially to its conception and content. Odysseas Papanikolaou contributed to the implementation and experimental results of this research. Johannes Betz revised the paper critically. Markus Lienkamp made an essential contribution to the conception of the research project. He revised the paper critically for important intellectual content. He gave final approval of the version to be published and agrees to all aspects of the work. As a guarantor, he accepts the responsibility for the overall integrity of the paper. We express gratitude to Continental Engineering Service for funding for the underlying research project.

\section{REFERENCES}

[1] J. Betz, A. Wischnewski, A. Heilmeier, F. Nobis, T. Stahl, L. Hermansdorfer, and M. Lienkamp, "A software architecture for an autonomous racecar," in 2019 IEEE 89th Vehicular Technology Conference (VTC2019-Spring). IEEE, 2019, pp. $1-6$.

[2] Infotip Service GmbH, "Globale navigations-satellitensysteme - gnss," 2019. [Online]. Available: https://kompendium.infotip. de/id-4-fehlerquellen-erweiterungen-und-verbesserungen.html

[3] R. Mur-Artal and J. D. Tardos, "Orb-slam2: An open-source slam system for monocular, stereo, and rgb-d cameras," IEEE Transactions on Robotics, vol. 33, no. 5, pp. 1255-1262, 2017.

[4] E. Ward and J. Folkesson, "Vehicle localization with low cost radar sensors," in 2016 IEEE Intelligent Vehicles Symposium (IV). Piscataway, NJ: IEEE, 2016, pp. 864-870.

[5] G. Grisetti, C. Stachniss, and W. Burgard, "Improved techniques for grid mapping with rao-blackwellized particle filters," IEEE Transactions on Robotics, vol. 23, no. 1, pp. 34-46, 2007.

[6] S. Kohlbrecher, O. von Stryk, J. Meyer, and U. Klingauf, "A flexible and scalable slam system with full 3d motion estimation," in 2011 IEEE International Symposium on Safety, Security, and Rescue Robotics. IEEE, 2011, pp. 155-160.

[7] W. Hess, D. Kohler, H. Rapp, and D. Andor, "Real-time loop closure in 2d lidar slam," in 2016 IEEE International Conference on Robotics and Automation, Stockholm, Sweden, May 16th-21st, A. Okamura and A. Menciassi, Eds. Piscataway, NJ: IEEE, 2016, pp. 1271-1278.

[8] T. Stahl, A. Wischnewski, J. Betz, and M. Lienkamp, "Rosbased localization of a race vehicle at high-speed using lidar," E3S Web of Conferences, vol. 95, p. 04002, 2019.

[9] S. N. K. Rangan, V. G. Yalla, D. Bacchet, and I. Domi, "Improved localization using visual features and maps for autonomous cars," in 2018 IEEE Intelligent Vehicles Symposium $(I V)$. Piscataway, NJ: IEEE, 2018, pp. 623-629. 
[10] M. R. U. Saputra, A. Markham, and N. Trigoni, "Visual slam and structure from motion in dynamic environments," $A C M$ Computing Surveys, vol. 51, no. 2, pp. 1-36, 2018.

[11] V. Casser, S. Pirk, R. Mahjourian, and A. Angelova, "Depth prediction without the sensors: Leveraging structure for unsupervised learning from monocular videos." [Online]. Available: https://arxiv.org/abs/1811.06152

[12] I. Alhashim and P. Wonka, "High quality monocular depth estimation via transfer learning," 2018. [Online]. Available: https://arxiv.org/pdf/1812.11941

[13] C. Godard, O. M. Aodha, and G. J. Brostow, "Unsupervised monocular depth estimation with left-right consistency," in CVPR, 2017.

[14] J. Engel, V. Koltun, and D. Cremers, "Direct sparse odometry," IEEE transactions on pattern analysis and machine intelligence, 2018.

[15] A. Geiger, P. Lenz, and R. Urtasun, "Are we ready for autonomous driving? the kitti vision benchmark suite," in Conference on Computer Vision and Pattern Recognition (CVPR), 2012.

[16] N. Yang, R. Wang, X. Gao, and D. Cremers, "Challenges in monocular visual odometry: Photometric calibration, motion bias and rolling shutter effect," 2017. [Online]. Available: https://arxiv.org/pdf/1705.04300

[17] R. Kümmerle, G. Grisetti, H. Strasdat, K. Konolige, and W. Burgard, "G2o: A general framework for graph optimization," in 2011 IEEE International Conference on Robotics and Automation, May 2011, pp. 3607-3613.

[18] E. Rublee, V. Rabaud, K. Konolige, and G. Bradski, "Orb: An efficient alternative to sift or surf," in 2011 International Conference on Computer Vision. IEEE, 2011, pp. 2564-2571.

[19] E. Karami, S. Prasad, and M. S. Shehata, "Image matching using sift, surf, brief and orb: Performance comparison for distorted images," CoRR, vol. abs/1710.02726, 2017.

[20] D. Galvez-López and J. D. Tardos, "Bags of binary words for fast place recognition in image sequences," IEEE Transactions on Robotics, vol. 28, no. 5, pp. 1188-1197, 2012.

[21] Jan Brehmer, "Orb-slam2 ros enabled," 2019. [Online]. Available: https://gitlab.tu-berlin.de/breakdowncookie/ORB_SLAM2

[22] A. Geiger, P. Lenz, C. Stiller, and R. Urtasun, "Vision meets robotics: The kitti dataset," International Journal of Robotics Research (IJRR), 2013.

[23] J. Sturm, N. Engelhard, F. Endres, W. Burgard, and D. Cremers, "A benchmark for the evaluation of rgb-d slam systems," in Proc. of the International Conference on Intelligent Robot Systems (IROS), Oct. 2012.

[24] D. DeTone, T. Malisiewicz, and A. Rabinovich, "Toward geometric deep slam," 2017/07/24. [Online]. Available: https: //arxiv.org/pdf/1707.07410

[25] E. Marti, M. A. de Miguel, F. Garcia, and J. Perez, "A review of sensor technologies for perception in automated driving," IEEE Intelligent Transportation Systems Magazine, vol. 11, no. 4, pp. 94-108, 2019.

\section{APPENDIX}

In the paper we presented the relative error of the localization module to the SLAM map. The global localization errors towards the ground truth are in general higher than the relative errors, due to addition of the errors arising during the map creation. The global error is presented in Table $\mathrm{V}$. To reduce the global error further, a more accurate map needs to be generated in the SLAM process. This could be achieved by fusing additional sensor data during the SLAM to stabilize the trajectory estimate e.g. through the use of GNSS systems. This is an approach widely considered in the automotive industry, given that a variety of sensor systems is fused for autonomous driving tasks [25].

TABLE V: Localization accuracy compared to ground truth

\begin{tabular}{|c|c|c|c|c|c|c|}
\hline Seq. & Mode & $\begin{array}{c}\text { Speed } \\
(\mathrm{m} / \mathrm{s})\end{array}$ & $\begin{array}{c}L T \\
(\%)\end{array}$ & $\begin{array}{c}t_{r e l} \\
(\%)\end{array}$ & $\begin{array}{c}r_{\text {rel }} \\
(\% / 100 \mathrm{~m})\end{array}$ & $\begin{array}{c}t_{\text {abs }} \\
(\mathrm{m})\end{array}$ \\
\hline \hline 01 & SLAM & 10 & 0 & 1.31 & 0.2 & 9.89 \\
01 & LOC & 37 & 1.9 & 6.36 & 0.82 & 13.89 \\
01 & LOC & 42 & 6.62 & 9.51 & 0.92 & 15.24 \\
\hline 07 & SLAM & 6 & 0 & 0.51 & 0.29 & 0.52 \\
07 & LOC & 12 & 2.35 & 0.62 & 0.33 & 0.53 \\
07 & LOC & 18 & 5.87 & 0.7 & 0.37 & 0.56 \\
07 & LOC & 24 & 8.67 & 0.93 & 0.42 & 1.12 \\
\hline 09 & SLAM & 8 & 0 & 0.84 & 0.25 & 1.66 \\
09 & SLAM & 20 & Fails & - & - & - \\
09 & LOC & 20 & 0.147 & 1.01 & 0.53 & 1.71 \\
09 & LOC & 25 & 2.15 & 1.09 & 0.55 & 1.72 \\
09 & LOC & 36 & 7.44 & 1.27 & 0.58 & 1.8 \\
\hline
\end{tabular}

